\title{
COMPOSITE POLYSILICON-PLATINUM LATERAL NANOELECTROMECHANICAL RELAYS
}

\author{
R. Parsa, S. Chong, N. Patil, K. Akarvardar', J. Provine, D. Lee, D. Elata ${ }^{2}$, S. Mitra, H.-S. P. Wong, R. T. Howe \\ Center for Integrated Systems, Dept. of Electrical Engineering, Stanford University, Stanford, CA 94305 \\ ${ }^{1}$ SEMATECH, Albany, NY, USA $\quad{ }^{2}$ Technion - Israel Institute of Technology, Haifa, Israel
}

\begin{abstract}
This paper reports the fabrication and performance of laterally actuated, polysilicon-platinum composite nanoelectromechanical (NEM) relays. Laterally actuated relays are defined in a single lithography step, enabling symmetric electrode and beam structures. The platinum coating serves as the conducting contact material and can also provide a local routing layer. Decoupling mechanical and electrical properties of the NEM relay allows independent optimization of each property. The NEM relays exhibited less than $3 \mathrm{k} \Omega$ contact resistance and can operate for $10^{8}$ cycles in room ambient.
\end{abstract}

\section{INTRODUCTION}

In advanced CMOS technology nodes, the minimum static power dissipation is limited by finite subthreshold slope and gate leakage. NEM relays are promising devices for low power logic applications due to zero leakage current, infinite subthreshold slope, and scalable actuation voltage [1,2]. SRAM cells [3] and FPGA blocks, where low switching speed and limited number of cycles are acceptable [4], are two attractive near-term applications for NEM relays.

Polysilicon has been characterized extensively as a structural material for MEMS devices and is known to be an excellent elastic material for mechanical structures. However, it is not well suited for electrical contacts, due to the formation of native oxide on the surface of polysilicon when exposed to oxygen. Passing current through contacting polysilicon surfaces requires a high applied voltage between the two sides of the contact to break through the native oxide. The resulting high field modifies the surface of the contact after every switching event, causing variations in the device characteristics. Furthermore, the device performance degrades rapidly due to contact degradation, in-use stiction, or particle attraction.

Although all-metal switches usually mitigate the problem of native oxide formation and require smaller applied voltages across the contact to pass current; their long-term mechanical reliability has yet to be demonstrated. Ruthenium switches showed functionality with low biases across the contact and survived up to about $2 \times 10^{6}$ cycles in a sealed probe station at room pressure [5]. TiN switches actuated in oil demonstrated significant reliability improvement over operation in air ambient [6,7]. Tungsten switches fabricated via Atomic Layer Deposition (ALD) were operated at biases less than $1 \mathrm{~V}$ across the contact [8]. AlN switches utilizing piezoelectric actuation have also been recently demonstrated [9].

MEMS structures with metal coating have been demonstrated for various RF, optical and sensing applications. Detailed studies on various metal alloys as the electric contact for MEMS switches have been published in the past [10]. Large vertically actuated switches with metal-coated contacts covered with a thin dielectric [11] have shown more than $10^{9}$ cycle lifetimes in $\mathrm{N}_{2}$ ambient. Functionality of basic circuit elements have been demonstrated by relays fabricated via this method [1]. In this work, we use composite laterally actuated relays consisting of a polysilicon beam with Pt-coated sidewalls, which allows the mechanical and electrical contact reliability issues to be decoupled.

\section{PRINCIPLE OF OPERATION}

A prototypical three terminal relay consists of three parts: 1) a deflecting beam (or source), which forms the channel for current flow; 2) an actuating gate electrode, which exerts force to the beam and determines the state of the switch; and 3) a drain electrode, which contacts the beam in the on state. Fig. 1a shows a five terminal device with one beam, two symmetrical gates and drains placed on each side of the beam in an on state configuration.

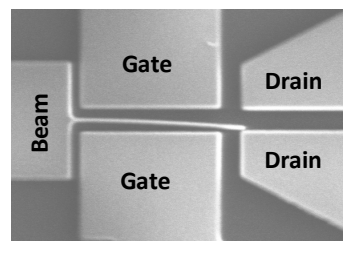

(a)

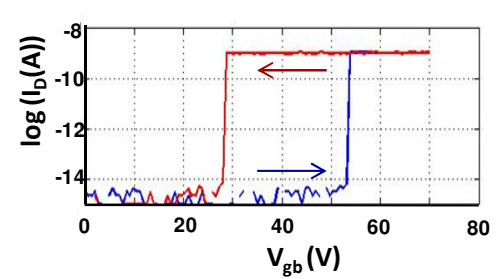

(b)
Figure 1. (a) SEM of a five terminal NEM relay with $14.2 \mu \mathrm{m}$-long beam and $(b)$ its hysteretic I-V characteristic. Due to the symmetry of the structure, identical I-V characteristics were observed when actuating to each side.

When $\left|V_{\text {gate }}-V_{\text {beam }}\right|\left(\left|V_{g b}\right|\right)$ is applied, the electrostatic force attracts the beam toward the gate, which is balanced by the elastic force of the deflected beam. Beyond the pull-in voltage $\left(\left|V_{g b}\right|>V_{P I}\right)$, elastic forces can no longer balance the electrostatic force, and the beam collapses toward the gate until contact is made at the drain. Since pull-in is achieved at an unstable state, the voltage at which the beam disconnects from the drain (pull-out voltage, $V_{P O}$ ) is smaller than $V_{P I}$, resulting in a hysteretic characteristic (Fig. 1b).

\section{DESIGN AND FABRICATION}

In designing lateral NEM relays, multiple parameters such as beam width $(t)$, beam length $(L)$, beam-to-gate gap $\left(g_{0}\right)$, beam-todrain gap $(d)$, beam-drain overlap $\left(L_{d}\right)$, drain-to-gate space $\left(S_{g d}\right)$, and beam-gate overlap $\left(L_{g}\right)$, are considered as shown in Fig. 2.

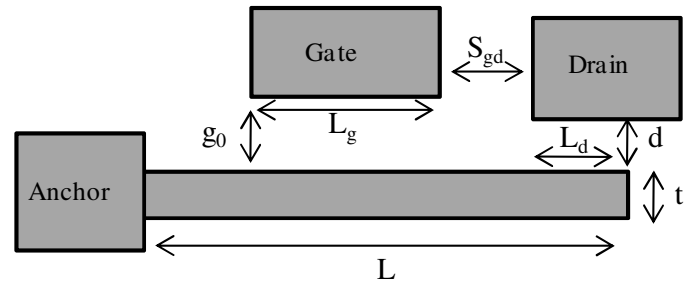

Figure 2. Schematic of a three terminal NEM relay and design parameters.

$V_{P I}$ and $V_{P O}$ can be controlled by adjusting the physical dimensions of the NEM relays. According to the 1-D parallel plate model [12], $V_{P I}$ and $V_{P O}$ are estimated as: 


$$
V_{p i}=\sqrt{\frac{8 k g_{0}^{3}}{27 \varepsilon W L}}, \quad V_{p o}=\sqrt{\frac{2 k}{\varepsilon W L} g_{\min }^{2}\left(g_{0}-g_{\min }\right)}, \quad k=\frac{2 E W t^{3}}{3 L^{3}}
$$

where $k$ is the spring constant of the beam depending on its geometry and material, $E$ is the Young's modulus of the beam material, $\varepsilon$ is the permittivity of the ambient enclosing the relay, (e.g. vacuum or oil), and $g_{\text {min }}$ is the minimum gap between gate and beam when beam is pulled in. In this model the fringing field effect is neglected. Further, it is assumed that the actuation electrode overlaps the entire length of the beam. It is important to allocate sufficiently large $S_{g d}$ to ensure the beam does not touch the gate after connecting to the drain. Unfortunately, for a fixed $L$ increasing $S_{g d}$ increases the actuation voltage due to the reduction in $L_{g}$, the length over which electrostatic force is exerted. Adequate amount of $L_{d}$ is required to ensure the beam touches the drain when pulled in.

It can be seen in Eq. (1) there are several parameters that can be adjusted to tune $V_{P I}$ such as the spring constant of the beam $(k)$ [13], dielectric constant $(\varepsilon)$ [7], and actuation gap $\left(g_{0}\right)$. One possible method of reducing $g_{0}$ is to deposit a material on the beam and electrode sidewalls. Although the increase in the beam width stiffens it, the decrease in the electrode gap dominates and leads to a decrease in the actuation voltage when the desired coating material with adequate thickness is used (Fig. 3).

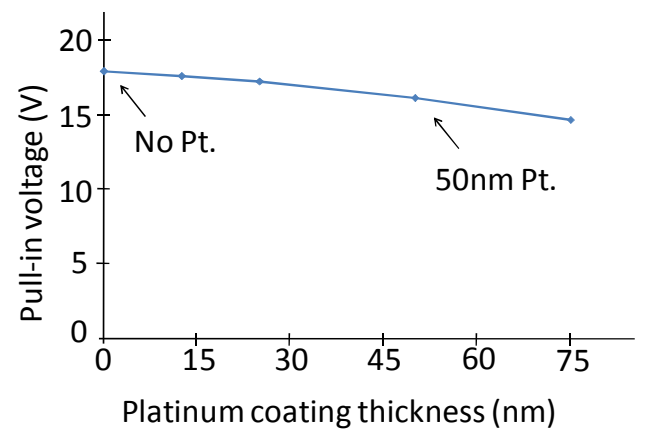

Figure 3. Pull-in voltage calculation vs. deposited sidewall metal on a $24 \mu \mathrm{m}$-long, 600nm-wide polysilicon beam with 500nm-wide actuation gap.

SEM images showing actuation of the device and the process steps for fabrication of the NEM relay are provided in Fig. 4a and $4 b$, respectively.

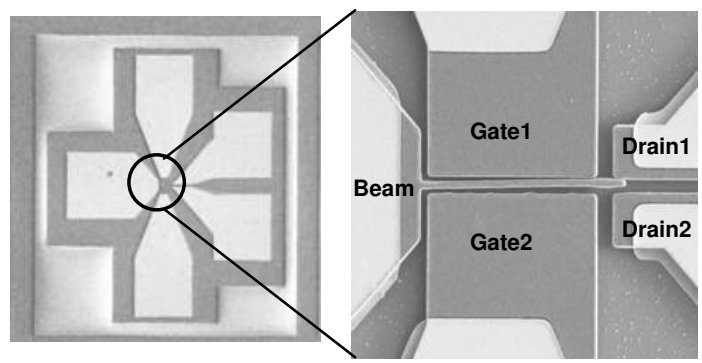

Figure 4a. SEM images showing actuation of a 5-terminal polysilicon switch coated with platinum on the sidewalls and selected top surfaces. Beam length=16 $\mu \mathrm{m}$, beam-drain overlap $=2 \mu \mathrm{m}$, gate-drain space $=2 \mu \mathrm{m}$, beam-to-gate gap $=600 \mathrm{~nm}$, beam-to-drain gap $=500 \mathrm{~nm}$.

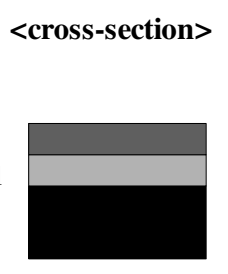

(b) Pattern polysilicon layer using 300nmLTO oxide hard mask
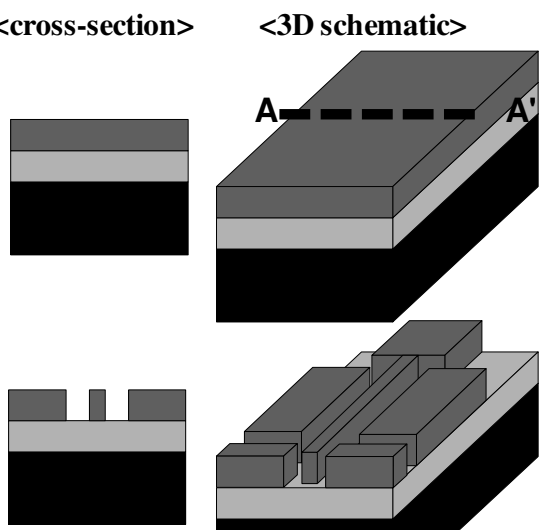

(c) Sputter $50 \mathrm{~nm}$ platinum
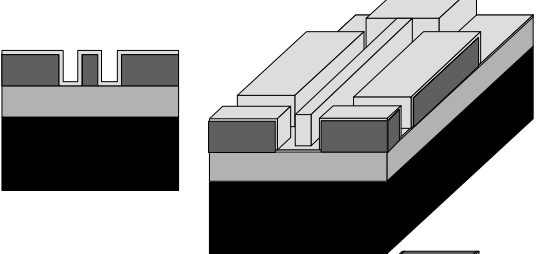

(d) Pt directional etch, leaving Pt on sidewalls only

(e) Pt liftoff, leaving Pt on pads
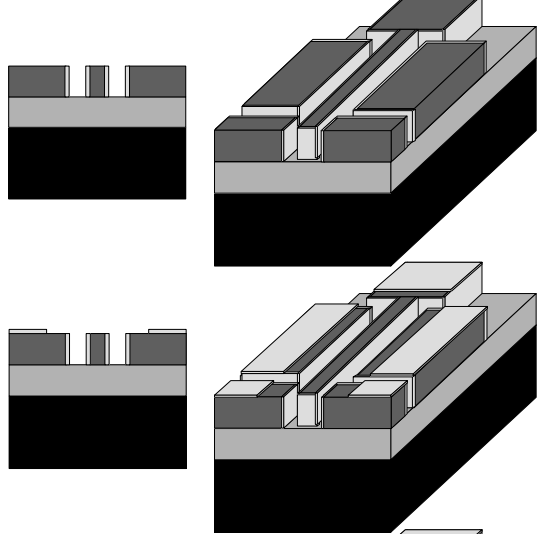

(f) Release in $49 \%$ HF followed by CPD
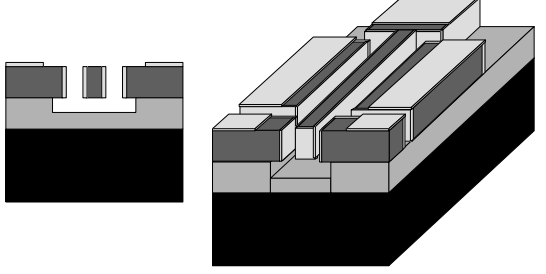

Figure $4 b .3 D$ and $2 D$ cross section view (taken along $A-A$ ' at all steps) of process flow used in fabrication of polysilicon relays with Pt sidewall coating (not drawn to scale).

First, a $2 \mu \mathrm{m}$-thick oxide is deposited at $400^{\circ} \mathrm{C}$ to serve as both the sacrificial layer and insulating layer between the device layer and $\mathrm{Si}$ wafer. Next a $1.2 \mu \mathrm{m}$-thick layer of phosphorous-doped polysilicon is deposited at $580^{\circ} \mathrm{C}$ as the structural layer and annealed at $1075^{\circ} \mathrm{C}$ for $30 \mathrm{sec}$. To assist in obtaining a vertical etch profile, $300 \mathrm{~nm}$ oxide is deposited at $400{ }^{\circ} \mathrm{C}$ to serve as a hard mask for etching the polysilicon. This oxide is patterned using an i-line stepper at its $500 \mathrm{~nm}$ resolution limit and then etched by RIE ( 250 mTorr, $50 \mathrm{sccm} \mathrm{CHF}_{3}, 20 \mathrm{sccm} \mathrm{CF}$, $100 \mathrm{sccm}$ Ar). The patterns are transferred to the polysilicon device layer by another RIE step (12 mTorr, $25 \mathrm{sccm} \mathrm{Cl}_{2}, 140 \mathrm{sccm} \mathrm{HBr}, 3 \mathrm{sccm} \mathrm{O}$ ). A 50nm Pt layer is sputtered on the wafer followed by an anisotropic argon 
sputter etch (12.5 mTorr, 100W, $15 \mathrm{sccm}$ Ar, 8 minutes) of the platinum to remove the Pt film from the bottom and top surfaces, leaving the sidewalls intact. Openings on the pads are patterned via conventional optical lithography. This step is followed by evaporation of 50nm thick platinum. The undesired platinum is removed via liftoff, leaving the metal on top of the pads only. The process is completed by releasing the devices in $49 \%$ HF followed by a critical point drying (CPD) step to avoid release stiction. Fig. 5 shows an SEM of the beam and electrode coverage by the deposited platinum after release.

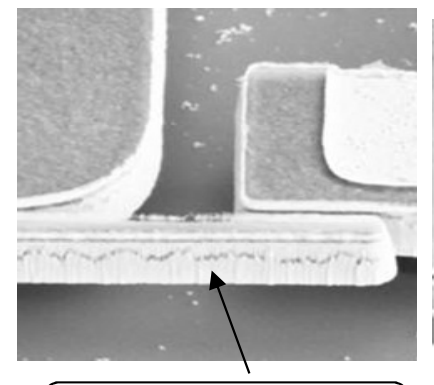

Sidewall roughness due to Pt sputter etch.

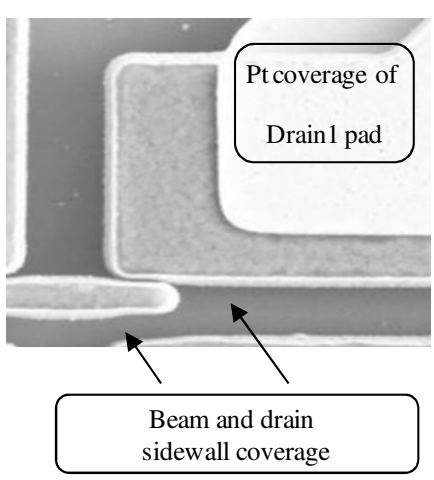
sidewall coverage
Figure 5. SEM image of polysilicon switch showing a continuous path from the beam-drain contact point to the top of the pad which will be probed.

\section{RESULTS}

Contact resistance was extracted by measuring the drain-tobeam current after pull-in under different drain biases. When tested in room-ambient immediately after the CPD step, the relays had a contact resistance of less than $3 \mathrm{k} \Omega$, for drain biases ranging from $10 \mathrm{mV}$ to $1 \mathrm{~V}$ (Fig. 6). In contrast, polysilicon NEM relays without coating had G $\Omega$-range contact resistance with drain biases ranging from $3 \mathrm{~V}$ to $5 \mathrm{~V}$. The Pt-coated relay's contact resistance is in the desired range for such relays because the mechanical delay typically is higher than RC delays [2]. Too low of a contact resistance will cause significant current flow after the switch closes and could cause overheating.

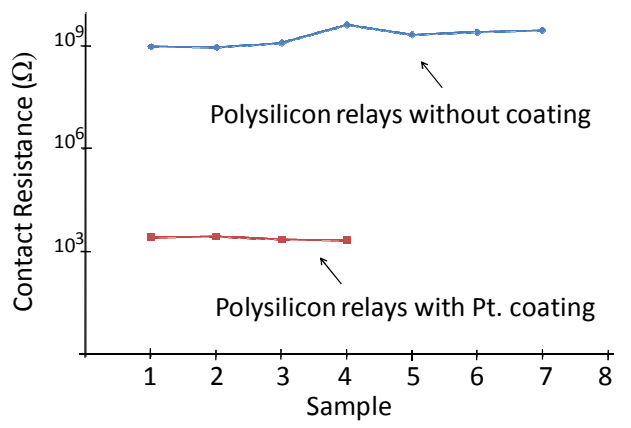

Figure 6. Pt-coated relays show $100 \mathrm{X}$ reduction in contact resistance $\left(0.01 V<V_{D}<1 V\right)$ in comparison to non-coated relays $\left(3 V<V_{D}<5 V\right)$. Devices were tested in room ambient immediately following release and drying.

The platinum film coating the beam and sidewalls reduces the actuation gap $\mathrm{g}_{0}$, which lowers the pull-in voltage of the relays as expected (Fig 7). Another method to reduce the actuation voltage is changing the environment surrounding the relay and increasing the dielectric constant of the material in the gap between the gate and the beam. Actuating fabricated switches in oil reduced the actuation voltage by a factor close to $\left(\varepsilon_{\mathrm{oil}} / \varepsilon_{\mathrm{air}}\right)^{0.5}$ and yields sharper pull-in and pull-out (Fig. 8). Repeated measurements of the same relay in oil yielded consistent actuation and contact characteristics.

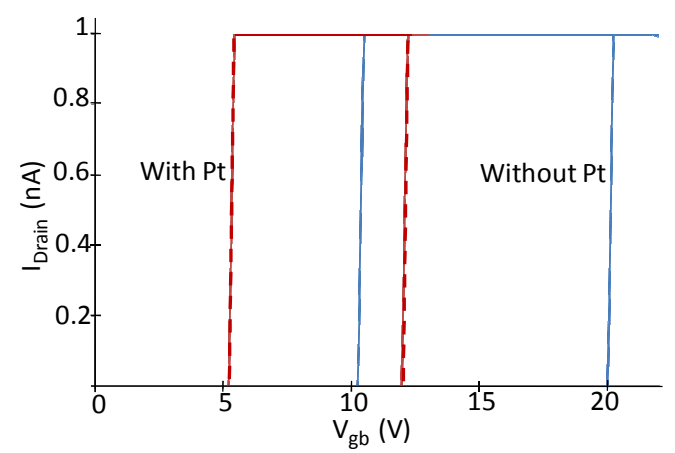

Figure 7. Experimental data showing reduced pull-in bias of a $22.5 \mu \mathrm{m}$-long polysilicon beam via 50nm Pt deposition. Compliance limit is set to $\ln A$.

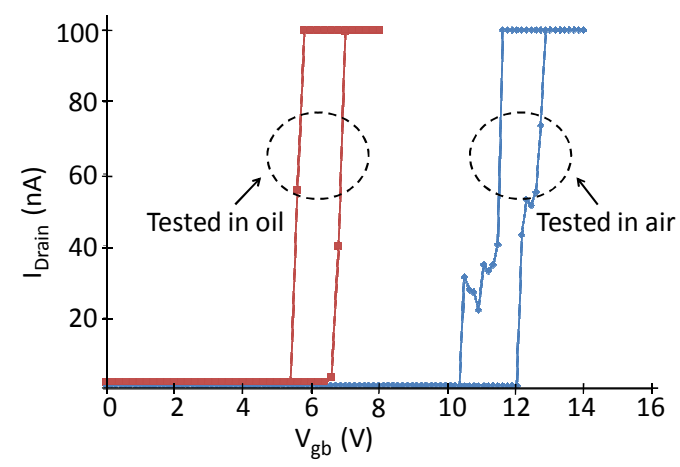

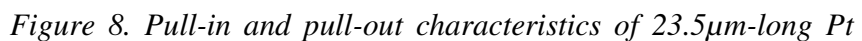
coated polysilicon switch in air and oil. Actuation bias is reduced in oil due to higher dielectric constant (1.8) of oil. Compliance limit is set to 100nA.

To observe the effect of electrical stress, contact-and-hold procedures were performed in room ambient on several coated NEM relays by passing up to $1 \mu \mathrm{A}$ current while in contact.

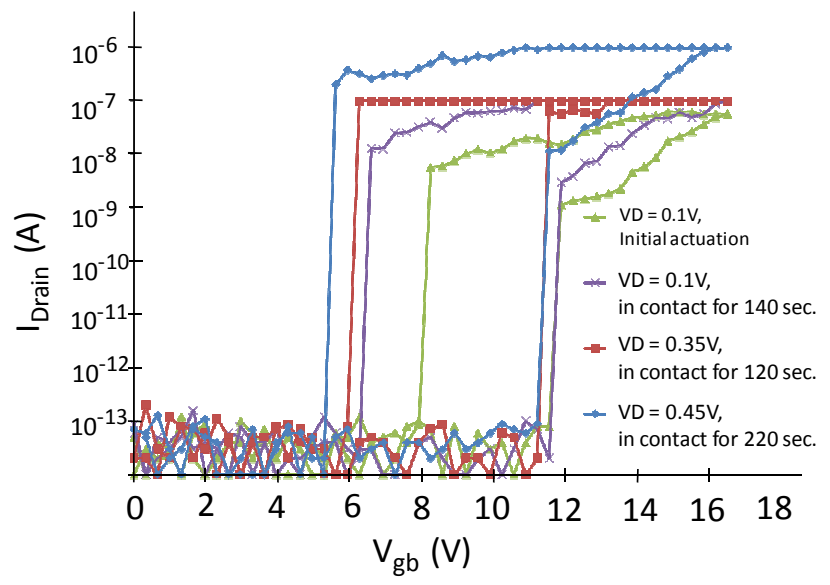

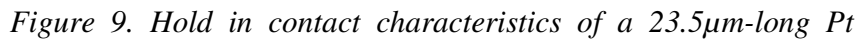
coated polysilicon beam showing reliable pull-in and pull-out after being in contact for more than $8 \mathrm{~min}$. The relay passes $\sim 0.25 \mathrm{mC}$ with drain biases of $0.1 \mathrm{~V}, 0.35 \mathrm{~V}$, and $0.45 \mathrm{~V}$ (with current compliances of $0.1 \mu \mathrm{A}, 0.1 \mu \mathrm{A}$, and $1 \mu \mathrm{A}$, respectively). 
No in-use stiction was observed during room-ambient operation. Reliable pull-in and pull-out were achieved after the beam was in contact with the drain for more than 8 minutes, with drain biases of $0.1 \mathrm{~V}$ to $0.45 \mathrm{~V}$, corresponding to approximately $0.25 \mathrm{mC}$ of charge passing through the contact (Fig. 9). An increase in contact resistance after many contacts was observed.

The NEM relay used for the contact-and-hold test (Fig. 9) was also subjected to cycling tests. Since the device was stored in dry nitrogen ambient for two days, first a pull-in and pull-out test was performed to ensure that the device is still functional. To cycle the device, the beam was connected to $0 \mathrm{~V}$ and drain to $100 \mathrm{mV}$ with a current compliance of $100 \mathrm{nA}$ on both terminals. The gate was then pulsed from $0 \mathrm{~V}$ to $16.5 \mathrm{~V}$. The pulse had a rise and fall time of $100 \mathrm{nsec}$. A few pulses of $1 \mathrm{~Hz}$ with duty cycle of $50 \%$ were applied multiple times to ensure the functionality of the device and setup. Next the frequency was changed to $250 \mathrm{kHz}$ and the current on the beam and drain was monitored. Analytical calculations of the fundamental resonant frequency for this relay yield a value of $860 \mathrm{kHz}$. Due to sampling limitations, currents were recorded every 5 sec. Device survived over $10^{8}$ switching cycles (Fig. 10).

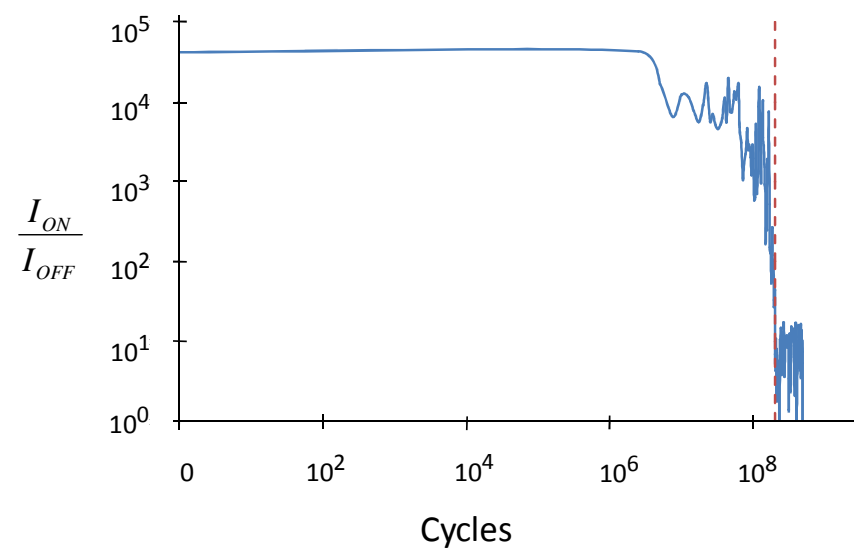

Figure 10. Endurance data operated in room ambient showing more than $10^{8}$ cycles with $I_{o n} / I_{\text {off }}>10^{3}$.

A sharp decrease of $\mathrm{I}_{\mathrm{ON}} / \mathrm{I}_{\mathrm{OFF}}$ after $2 \times 10^{8}$ cycles was observed. Hot switching of the relay after the contact-and-hold test could have caused the thin platinum coating to be damaged. Asperity size changes due to the test affects the contact resistance. Pull-in and pull-out test after completing $5 \times 10^{8}$ cycles showed the device was not functional. Testing of more devices in air, dry nitrogen, and oil environment will help to get a better understanding of the degradation mechanisms.

\section{CONCLUSION}

Laterally actuated NEM relays consisting of platinum-coated polysilicon composite beams were fabricated and demonstrated to have $<3 \mathrm{k} \Omega$ contact resistance and to operate for over $10^{8}$ switching cycles in room ambient conditions. The need for a single structural material having both proper electrical and mechanical properties has been eliminated through the use of the composite lateral relay design.

\section{ACKNOWLEDGEMENT}

Fabrication work was performed at the Stanford Nanofabrication Facility (a member of the National Nanotechnology Infrastructure Network) supported by the NSF under Grant ECS-9731293, its lab members, and the industrial members of the Stanford Center for Integrated Systems. This work was supported by the DARPA MTO program "Nano Electro Mechanical Computers (NEMS)" (Contract number: $\mathrm{NBCH}$ 1090002, Program manager: Dr. T. Akinwande). The authors would like to thank Nathan Klejwa, Scott Lee, Chen Chen, and Vivian Feig for helpful discussions.

\section{REFERENCES}

[1] F. Chen, M. Spencer, R. Nathanael, C. Wang, H. Fariborzi, A. Gupta, H. Kam, V. Pott, J. Jeon, T.-J. King Liu, D. Markovic, V. Stojanovic, and E. Alon, "Demonstration of Integrated Micro-Electro-Mechanical Switch Circuits for VLSI Applications", IEEE ISSCC 2010, pp. 150-152.

[2] K. Akarvardar, D. Elata, R. Parsa, G. C. Wan, K. Yoo, J Provine, P. Peumans, R. T. Howe, and H.-S. P. Wong, "Design Considerations for Complementary Nanoelectromechanical Logic Gates", IEDM 2007, pp. 299-302.

[3] S. Chong, K. Akarvardar, R. Parsa, J.-B. Yoon, R. T. Howe, S. Mitra, and H.-S. P. Wong, "Nanoelectromechanical (NEM) Relays Integrated with CMOS SRAM for Improved Stability and Low Leakage", ICCAD 2009, pp. 478-484.

[4] C. Chen, R. Parsa, N. Patil, S. Chong, K. Akarvardar, J. Provine, D. Lewis, J. Watt, R. T. Howe, H.-S. P. Wong, and S. Mitra, "Efficient FPGAs using Nanoelectromechanical Relays", ACM Intl. Symp. FPGA 2010, pp. 273-282.

[5] D. A. Czaplewski, G. A. Patrizi, G. M. Kraus, J. R. Wendt, C. D. Nordquist, S. L. Wolfley, M. S. Baker, and M. P. de Boer "A Nanoelectromechanical Switch for Integration with CMOS Logic", Journal of Micromechanics and Microengineering, vol. 19, 2009 .

[6] W. W. Jang, J.-B. Yoon, M.-S. Kim, J.-M. Lee, S.-M. Kim, E.-J. Yoon, K. H. Cho, S.-Y. Lee, I.-H. Choi, D.-W. Kim, and D. Park, "NEMS switch with 30 nm-thick beam and 20 nm-thick air-gap for high density non-volatile memory applications", Solid State Electronics 2008, pp. 1578-1583.

[7] J.-O. Lee, M.-W. Kim, S.-D. Ko, H.-O. Kang, W.-H. Bae, M.H. Kang, K.-N. Kim, D.-E. Yoo, and J.-B. Yoon, "3-T Nanoelectromechanical Switching Device in Insulating Liquid Media for Low Voltage Operation and Reliability Improvement", IEDM 2009, pp. 227-230.

[8] B.D. Davidson, S.M. George, and V.M. Bright, "Atomic layer deposition (ALD) Tungsten nanoelectromechanical transistors", Proc. IEEE MEMS 2010, pp. 424-427.

[9] N. Sinha, T. Jones, Z. Guo, and G. Piazza, "Demonstration of low voltage and functionally complete logic operations using body-biased complementary and ultra-thin AlN piezoelectric mechanical switches", Proc. IEEE MEMS 2010, pp. 751-754.

[10] R. A. Coutu, Jr, P. E. Kladitis, K. D. Leedy, and R. L. Crane, "Selecting metal alloy electric contact materials for MEMS switches", Journal of Micromechanical Microengineering, 2004, 1157-1164.

[11] H. Kam, V. Pott, R. Nathanael, J. Jeon, E. Alon, and T.-J. King Liu "Design and Reliability of a Micro-Relay Technology for Zero-Standby-Power Digital Logic Applications", IEDM 2009, pp. 809-812.

[12] V. Kaajakari, Practical MEMS, Small Gear Publishing, 2009.

[13] D. Lee, W. S. Lee, J. Provine, J.-O. Lee, J.-B. Yoon, R. T. Howe, S. Mitra, and H.-S. P. Wong, "Titanium Nitride Sidewall Stringer Process for Lateral Nanoelectromechanical Relays”, Proc. IEEE MEMS 2010, pp. 456-459. 